07

\title{
Анализ ошибок записи и считывания голографической призмы на фото-термо-рефрактивном стекле
}

\author{
(C) Doan Van Bac, C.A. Иванов, Р.А. Окунь, А.Е. Ангервакс, Н.В. Никоноров \\ Университет ИТМО, \\ 197101 Санкт-Петербург, Россия \\ e-mail: vanbacdoan@yahoo.com.vn
}

Поступила в редакцию 09.02.2018 г.

Проведен анализ влияния отклонений условий записи и считывания мультиплексных брэгговских решеток от расчетных значений на параметры восстанавливаемого „веера“ лучей голографической призмы (ГП), сформированной брэгговскими решетками в фото-термо-рефрактивном стекле. Отклонения заданы либо как поворот образца вокруг одной из трех ортогональных осей $O x y z$, либо его одновременного поворота вокруг двух осей. Показано, что ошибки в установке образца при записи не оказывают влияния на качество ГП, если все решетки записаны с одинаковым отклонением от идеального положения. Однако, если при записи каждой решетки ошибки различны, это приводит к снижению плоскостности веера ГП и к потере ее работоспособности. Рассчитаны предельные значения ошибок поворотов вокруг осей $O x$ и $O y$, позволяющие сохранить функциональность ГП, т.е. одновременное восстановление всех ее лучей. Установлено, что в случае идеально записанной ГП ошибки на этапе считывания могут приводить к скручиванию веера и нарушению его плоскостности.

DOI: $10.21883 /$ OS.2018.06.46091.37-18

\section{Введение}

В работах [1-3] был предложен новый оптический элемент - голографическая призма (ГП), созданный на основе аддитивно окрашенных кристаллов фторида кальция. При воздействии на ГП референтного лазерного луча индуцируется отклик в виде нескольких дифрагированных лучей. В зависимости от способа записи они могут возникать поочередно при повороте образца (I модификация) или одновременно (II модификация) [1], охватывая ограниченный диапазон углов („веер“ лучей). На основе ГП могут быть созданы углоизмерительные/углозадающие приборы, удовлетворяющие трем противоречивым требованиям - мобильность, высокая дискретность и точность угловых измерений. Однако применение фторида кальция в качестве голографической среды выявило ряд существенных недостатков: необходимость в большой энергии экспозиции при записи голограмм, малый динамический диапазон изменения показателя преломления и наличие поглощения в видимом диапазоне спектра. Применение фото-терморефрактивного (ФТР) стекла в качестве голографической среды для создания ГП позволяет не только избавиться от недостатков флюорита, но и улучшить параметры ГП [4,5].

В настоящей работе рассматривается влияние отклонений условий записи и считывания ГП от расчетных на такие параметры „веера“, как угловое расстояние между его лучами, угол поворота каждой решетки внутри среды, угол считывания, угол, под которым луч выходит из ГП, и плоскостность веера.

\section{1. Параметры голографической призмы и условия расчета}

Голографическая призма представляет собой плоскопараллельный образец ФТР стекла, в объеме которого записано несколько наложенных голограмм (решеток) таким образом, что их считывание происходит одновременно, однако каждая восстанавливает луч под своим углом $\beta(i)$ к поверхности элемента. Таким образом, при считывании на расчетной длине волны под углом $\alpha_{0}$ возникает „веер“ лучей, при этом угол $\beta(i)$ для каждого луча определяется следующим соотношением: $\beta(i)=\beta_{0}+i \chi$, где $\beta_{0}-$ угол дифракции первого луча, $\chi-$ угловое расстояние между соседними лучами веера, $i$ - номер луча $(0,1,2, \ldots)$. При создании ГП для каждого луча „веера“ необходимо записать отдельную решетку с периодом $d(i)$, повернутую внутри образца на угол $\phi(i)$. Указанные параметры определяются на этапе расчета призмы исходя из требуемых значений $\alpha_{0}$ и $\beta(i)$ согласно следующим соотношениям:

$$
\begin{gathered}
d(i)=\frac{\lambda}{2 n \sin \left(\theta_{B}(i)\right)}, \\
\phi(i)=\arcsin \left(\frac{\sin \left(\alpha_{0}\right)}{n}\right)-\theta_{B}(i),
\end{gathered}
$$

где $n$ - показатель преломления среды, $\lambda$ - длина волны считывания.

При этом угол Брэгга $\left(\theta_{B}(i)\right)$ для $i$-й решетки в призме определяется как:

$$
\theta_{B}(i)=\frac{\arcsin \left(\frac{\sin \alpha_{0}}{n}\right)+\frac{\beta(i)}{|\beta(i)|}\left|\arcsin \left(\frac{\sin \beta(i)}{n}\right)\right|}{2} .
$$



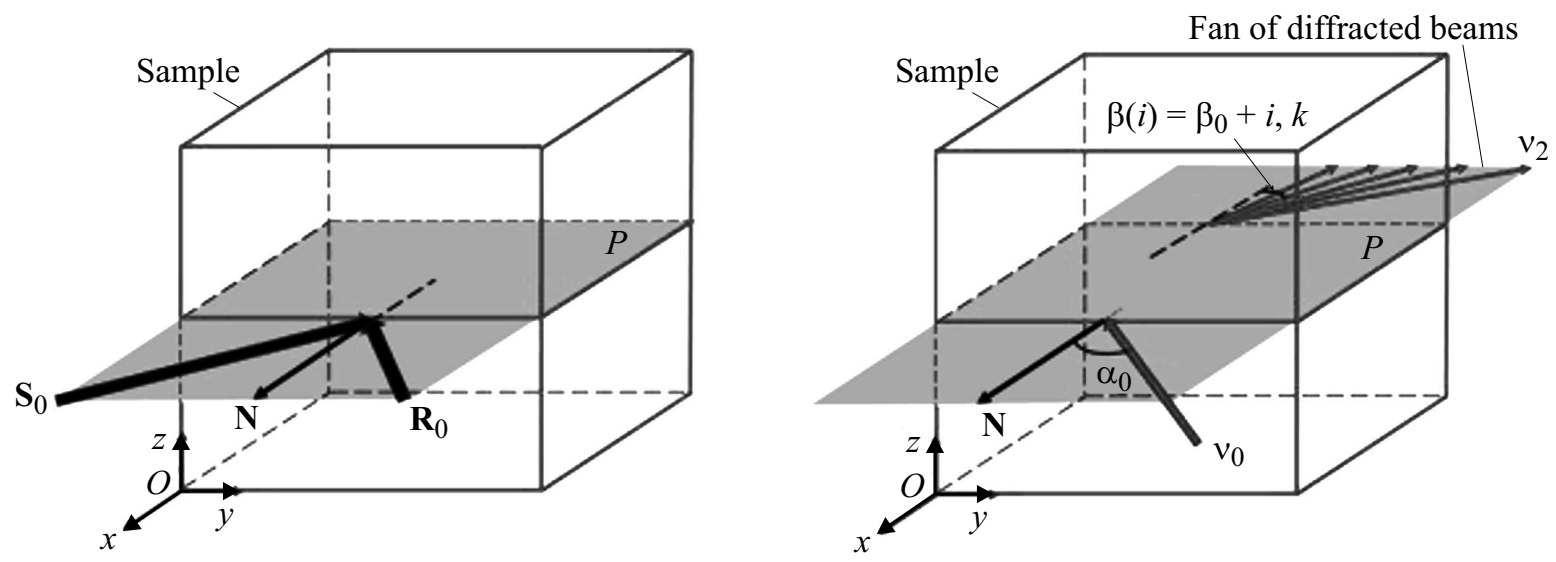

C
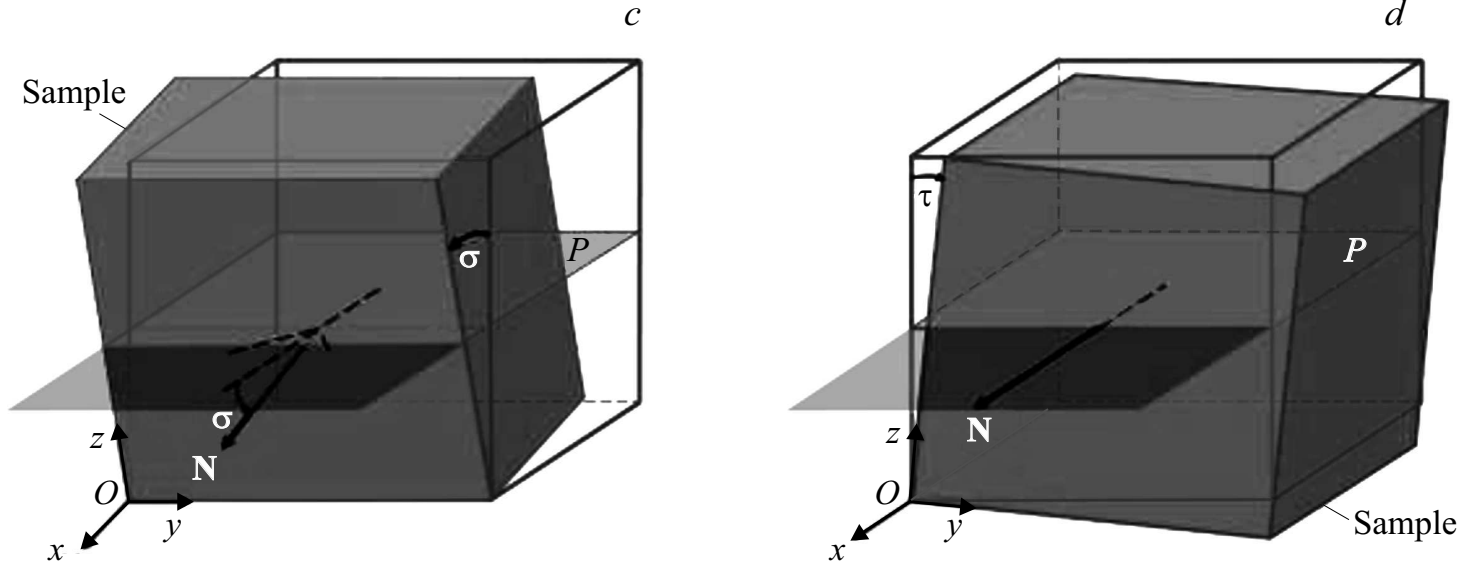

Рис. 1. Положение плоскости записи $P$ относительно координатной плоскости $O y z$, связанной с рабочей поверхностью ГП $(a, b-$ идеальный случай; $c-$ поворот ГП вокруг оси $O y$ на угол $\sigma ; d-$ поворот ГП вокруг оси $O x$ на угол $\tau)$.

Следует отметить, что дальнейший расчет проводится с учетом особенностей экспериментальной установки, используемой в работе по записи ГП на ФТР стекле [6]. Положим, что в идеальном случае запись и считывание происходят в плоскости $P$ (рис. $1, a, b)$. Однако возможные ошибки в установке образца как на этапе записи, так и считывания ведут к изменению положения плоскости $P$ относительно координатной плоскости $O y z$, связанной с рабочей поверхностью элемента (рис. $1, c, d$ ), и соответственно к изменению в ориентации записывающих $\mathbf{S}_{0}, \mathbf{R}_{0}$ и считывающего $\mathbf{v}_{0}$ лучей.

В разд. 2 будет проанализирован поворот плоскости $P$ вокруг осей $O y$ (рис. 1,c) и $O x$ (рис. 1,d). При этом поворот для всех решеток ГП будет осуществляться на один и тот же угол. Также будет рассмотрен случай, когда решетки в составе ГП имеют различные ошибки в ориентации.

В разд. 3 будет рассмотрен одновременный поворот ГП вокруг двух ортогональных осей $O x$ и $O y$.

Во всех расчетах при повороте вокруг оси $O y$ будет рассмотрен интервал значений углов $\sigma= \pm 0.05^{\circ}$, который определяется погрешностью измерения дан- ного отклонения в экспериментальной схеме записи/считывания. Поворот вокруг оси $O x$ будет рассмотрен в интервале углов $\tau= \pm 2^{\circ}$, так как он контролируется существенно хуже. Поворот вокруг оси $\mathrm{Oz}$ рассматриваться не будет, так как он контролируется с достаточно высокой точностью $(0.36 \mathrm{~s})$.

\section{2. Влияние ошибок при повороте голографической призмы вокруг осей $O x$ и $O y$}

\section{1. Влияние ошибок при записи на угол между записывающими лучами в среде}

Здесь и далее рассмотрим реальную ГП, состоящую из 21 наложенной голографической решетки, со следующими параметрами: показатель преломления среды $n=1.498$, длина волны записывающего лазеpa $\lambda_{z}=325 \mathrm{~nm}$, длина волны считывающего лазера $\lambda=532 \mathrm{~nm}$, угол падения считывающего луча $\alpha_{0}=25^{\circ}$, угол дифракции для первой решетки $\beta_{0}=3^{\circ}$, угловая разница между дифрагированными лучами $\chi=2.5^{\circ}$. 


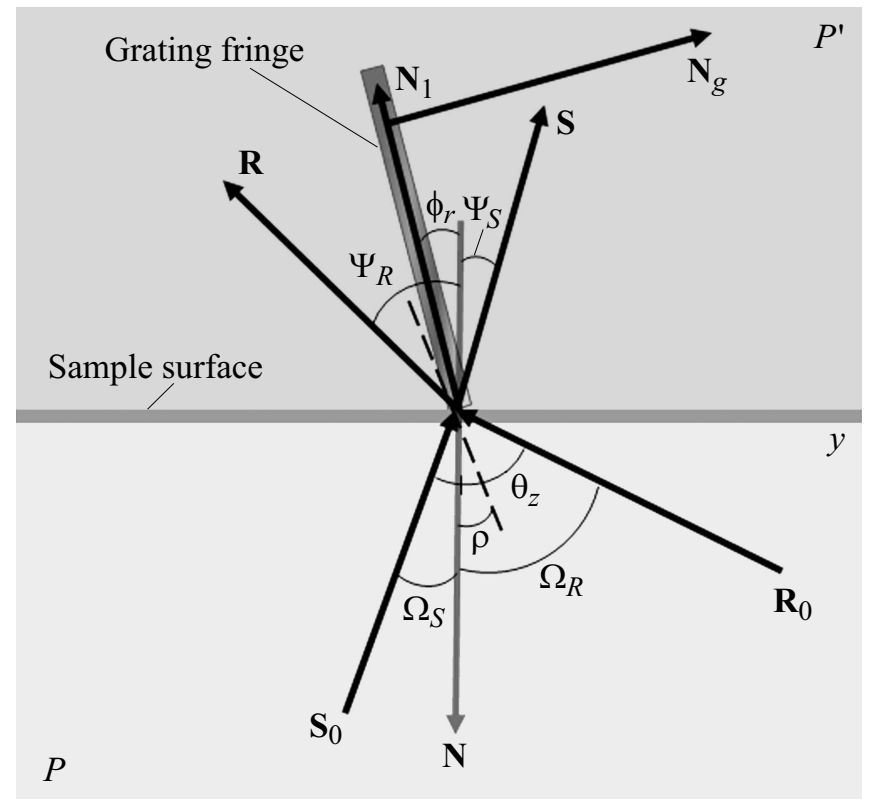

Рис. 2. Запись голографической решетки с плоскостями, повернутыми относительно нормали $\mathbf{N}$ к рабочей поверхности ГП на угол $\phi_{r}$.

Рассмотрим процесс записи более подробно (рис. 2). Записывающие лучи $\mathbf{S}_{0}$ и $\mathbf{R}_{0}$, лежащие в плоскости $P$, направляются на грань образца под углом $\Omega_{S}=\theta z-\rho$ и $\Omega_{R}=\theta z+\rho$ соответственно [6] и, преломляясь в среде под углами $\Psi_{S}$ и $\Psi_{R}$, образуют голограмму с вектором решетки $\mathbf{N}_{g}$ и углом поворота плоскостей пучности $\phi_{r}$ (рис. 2). Векторы $\mathbf{S}, \mathbf{R}$ и $\mathbf{N}_{g}$ лежат в плоскости $P^{\prime}$.

Направление векторов записывающих лучей $\mathbf{S}_{0}$ и $\mathbf{R}_{0}$ $i$-й решетки в воздухе в идеальном случае равно

$$
\begin{gathered}
\mathbf{S}_{0}(i)=\left(\begin{array}{c}
-\cos [\theta z(i)-\rho(i)] \\
\sin [\theta z(i)-\rho(i)] \\
0
\end{array}\right), \\
\mathbf{R}_{0}(i)=\left(\begin{array}{c}
-\cos [\theta z(i)+\rho(i)] \\
-\sin [\theta z(i)+\rho(i)] \\
0
\end{array}\right) .
\end{gathered}
$$

Поскольку мы связали систему координат с плоскостью образца, вектор нормали к рабочей поверхности ГП не зависит от ошибок ее установки. Примем, что наклон образца назад (поворот образца вокруг оси $O y$ по часовой стрелке) означает положительный угол $\sigma>0$, а поворот образца вокруг оси $O x$ против часовой стрелки означает положительный угол $\tau>0$ (рис. $1, c, d$ ). Положение векторов записывающих лучей относительно плоскости $O y z$ при поворотах относительно осей $O x$ и $O y$ можно найти как:

$$
\mathbf{R}_{0}(i) \equiv M \cdot \mathbf{R}_{0}(i), \quad \mathbf{S}_{0}(i) \equiv M \cdot \mathbf{S}_{0}(i),
$$

где $M$ - матрица поворота, которая определяется как [7]

$$
M=\left(\begin{array}{ccc}
1 & 0 & 0 \\
0 & \cos (\tau) & -\sin (\tau) \\
0 & \sin (\tau) & \cos (\tau)
\end{array}\right)
$$

при повороте вокруг оси $O x$ и

$$
M=\left(\begin{array}{ccc}
\cos (\sigma) & 0 & \sin (\sigma) \\
0 & 1 & 0 \\
-\sin (\sigma) & 0 & \cos (\sigma)
\end{array}\right)
$$

при повороте вокруг оси $O y$.

Так как направление вектора каждого записывающего луча меняется относительно координатной плоскости $O y z$, значение углов падения записывающих лучей также будет меняется согласно следующим соотношениям:

$\Omega_{R}(i)=\arccos \left[\frac{\mathbf{R}_{0}(i) \cdot \mathbf{N}}{\left|\mathbf{R}_{0}(i)\right||\mathbf{N}|}\right], \quad \Omega_{S}(i)=\arccos \left[\frac{\mathbf{S}_{0}(i) \cdot \mathbf{N}}{\left|\mathbf{S}_{0}(i)\right||\mathbf{N}|}\right]$.

Соответственно происходят изменения в углах преломления записывающих лучей в стекле, которые можно найти как

$\Psi_{R}(i)=\arcsin \left\{\frac{\sin \left[\Omega_{R}(i)\right]}{n}\right\}, \quad \Psi_{S}(i)=\arcsin \left\{\frac{\sin \left[\Omega_{S}(i)\right]}{n}\right\}$.

Таким образом, при отклонении положения образца в процессе записи от идеального направления векторов записывающих лучей в стекле будут задаваться следующими соотношениями:

$$
\begin{aligned}
\mathbf{R}(i)= & -\cos \left[\Psi_{R}(i)\right] \cdot \mathbf{N} \\
& +\sin \left[\Psi_{R}(i)\right] \frac{\mathbf{R}_{0}(i)+\cos \left[\Psi_{R}(i)\right] \cdot \mathbf{N}}{\left|\mathbf{R}_{0}(i)+\cos \left[\Psi_{R}(i)\right] \cdot \mathbf{N}\right|}, \\
\mathbf{S}(i)= & -\cos \left[\Psi_{S}(i)\right] \cdot \mathbf{N} \\
& +\sin \left[\Psi_{S}(i)\right] \frac{\mathbf{S}_{0}(i)+\cos \left[\Psi_{S}(i)\right] \cdot \mathbf{N}}{\left|\mathbf{S}_{0}(i)+\cos \left[\Psi_{S}(i)\right] \cdot \mathbf{N}\right|} .
\end{aligned}
$$

Следовательно, угол между записывающими лучами для $i$-й решетки в среде будет определяться как

$$
\theta(i)=\frac{1}{2} \arccos \left[\frac{\mathbf{R}(i) \cdot \mathbf{S}(i)}{|\mathbf{R}(i)||\mathbf{S}(i)|}\right] .
$$

\section{2. Влияние ошибок при записи на ориентацию решетки внутри голографической призмы}

Положение голографической плоскости в объеме ГП однозначно определяется направлением вектора решетки. Голографические плоскости делят пополам угол между волновыми векторами интерферирующих волн [8], 


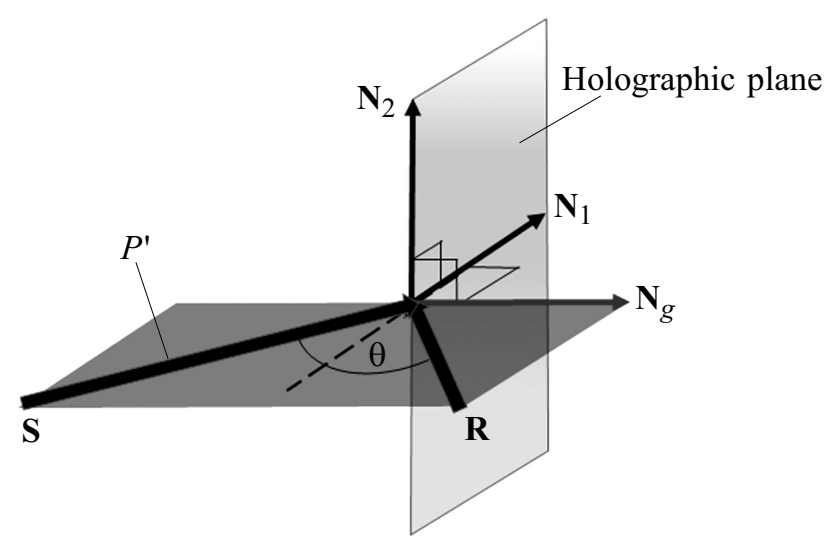

Рис. 3. Ориентация голографической плоскости внутри среды.

ориентацию вектора решетки $\left(\mathbf{N}_{g}\right)$ внутри среды можно определить через векторное произведение вектора биссектрисы $\left(\mathbf{N}_{1}\right)$ угла $\theta(i)$ и нормалью $\left(\mathbf{N}_{2}\right)$ к плоскости, образованной записывающими лучами в среде (рис. 3 ):

$$
\mathbf{N}_{g}(i)=\frac{\mathbf{N}_{1}(i) \times \mathbf{N}_{2}(i)}{\left|\mathbf{N}_{1}(i) \times \mathbf{N}_{2}(i)\right|},
$$

где векторы $\mathbf{N}_{1}$ и $\mathbf{N}_{2}$ определяются следующим образом:

$$
\mathbf{N}_{1}(i)=\frac{\mathbf{R}(i)+\mathbf{S}(i)}{|\mathbf{R}(i)+\mathbf{S}(i)|}, \quad \mathbf{N}_{2}(i)=\frac{\mathbf{S}(i) \times \mathbf{R}(i)}{|\mathbf{S}(i) \times \mathbf{R}(i)|} .
$$

Угол поворота голографических плоскостей каждой решетки в таком случае определяется как дополнительный угол к углу между вектором решетки и нормалью к входной грани образца:

$$
\phi_{r}(i)=90-\arccos \left[\frac{-\mathbf{N} \cdot \mathbf{N}_{g}(i)}{|\mathbf{N}| \cdot\left|\mathbf{N}_{g}(i)\right|}\right] .
$$

Анализ показал, что поворот образца вокруг осей $O x$ и $O y$ в заданных диапазонах углов $\tau$ и $\sigma$ не оказывают существенного влияния на период $d(i)$ и угол $\phi_{r}(i)$ в решетке. Изменения периода решетки при максимальных отклонениях углов не превышают $\Delta d(i) \approx 3.7 \mathrm{pm}$, а угла поворота голографических плоскостей голограммы $\Delta \phi(i) \approx 2 \cdot 10^{-5 \circ}$. Стоит отметить, что данная оценка предполагает, что векторы всех решеток лежат в одной плоскости, однако сама плоскость может поворачиваться в среде, что приведет к изменению угла считывания $\alpha_{0}$ и углов $\beta(i)$, под которым дифрагированные лучи выходят из образца.

\section{3. Влияние ошибок считывания на параметры голографической призмы}

Если все векторы решеток лежат в одной плоскости, то всегда найдется считывающий луч, удовлетворяющий условию Брэгга всех решеток одновременно. Поскольку ГП работает в видимом диапазоне спектра, найти такой луч или положение ГП не составляет труда.
Однако ГП будет работать корректно лишь тогда, когда считывающий луч лежит в той же плоскости, что и векторы всех записанных решеток. Рассмотрим ситуацию, когда считывающий луч удовлетворяет условию Брэгга всех решеток, но не лежит в одной плоскости с векторами всех записанных решеток.

При считывании голограммы считывающий луч $\mathbf{v}_{0}$ направляется на образец под углом $\alpha(i)$ и лежит в плоскости $O x y$ (рис. 4). При переходе в образец считывающий луч преломляется под углом $\alpha_{g}$, меняет свое направления на $\mathbf{v}_{1}$ и падает на решетку под углом $\theta_{B r}$. Возникающий при дифракции луч $\mathbf{v}_{1}^{\prime}$ падает на грань образца под углом $\beta_{g}$ и выходит в виде вектора $\mathbf{v}_{2}$ из образца под углом $\beta_{r}$.

В идеальном случае направление вектора считывающего луча равно

$$
v_{0}(\alpha(i))=\left(\begin{array}{c}
-\cos [\alpha(i)] \\
-\sin [\alpha(i)] \\
0
\end{array}\right) .
$$

Аналогично случаю записи направление вектора считывающего луча в реальном случае можно найти с использованием матриц поворота $M$. Таким образом, при переходе в регистрирующую среду получим следующее выражение для направления вектора $\mathbf{v}_{1}$ :

$$
\mathbf{v}_{1}(i)=-\cos \left(\alpha_{g}\right) \cdot \mathbf{N}+\sin \left(\alpha_{g}\right) \frac{\mathbf{v}_{0}(\alpha(i))+\cos [\alpha(i)] \cdot \mathbf{N}}{\left|\mathbf{v}_{0}(\alpha(i))+\cos [\alpha(i)] \cdot \mathbf{N}\right|}
$$

Тогда условие Брэгга можно записать в виде

$$
\theta_{B r}(i)=90^{\circ}-\arccos \left(\frac{-\mathbf{v}_{1}(i) \cdot \mathbf{N}_{g}(i)}{\left|\mathbf{v}_{1}(i)\right| \cdot\left|\mathbf{N}_{g}(i)\right|}\right),
$$

где $\theta_{B r}$ - угол считывания (угол Брэгга) $i$-й решетки.

Решая данное уравнение, можно найти угол $\alpha(i)$, под которым надо направлять считывающий луч на элемент при считывании $i$-й решетки.

При этом направление вектора дифрагированного луча $i$-й решетки в стекле можно найти как

$$
\mathbf{v}_{1}^{\prime}(i)=2 \cos \left[90^{\circ}-\theta_{B r}(i)\right] \cdot \mathbf{N}_{g}(i)+\mathbf{v}_{1}(i) .
$$

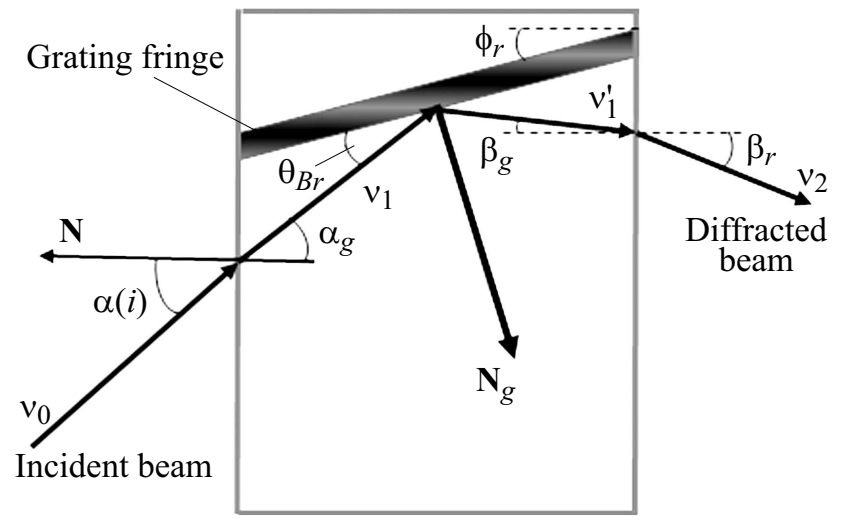

Pис. 4. Направление векторов считывающего и дифрагированного лучей при считывании голограммы. 
Далее найдем угол, под которым излучение $\mathbf{v}_{2}$ падает на грань образца в среде:

$$
\beta_{g}(i)=\arccos \left(\frac{-\mathbf{v}_{2}(i) \cdot \mathbf{N}}{\left|\mathbf{v}_{2}(i)\right| \cdot|\mathbf{N}|}\right)
$$

Тогда при переходе в воздух

$$
\beta_{r}(i)=\arcsin \left[n \sin \left(\beta_{g}(i)\right)\right] .
$$

Направление вектора дифрагированного луча в воздухе можно найти как

$$
\begin{aligned}
\mathbf{v}_{2}(i)= & -\cos \left[\beta_{r}(i)\right] \cdot \mathbf{N}+\sin \left[\beta_{r}(i)\right] \\
& \times \frac{\mathbf{v}_{1}^{\prime}(\alpha(i))+\cos \left[\beta_{g}(i)\right] \cdot \mathbf{N}}{\left|\mathbf{v}_{1}^{\prime}(\alpha(i))+\cos \left[\beta_{g}(i)\right] \cdot \mathbf{N}\right|} .
\end{aligned}
$$

Первым важным параметром ГП является угловая разница между соседними дифрагированными лучами $\chi(i)$. Этот параметр задается при расчете призмы, и ошибка в установке образца относительно осей $O x$ и $O y$ в исследуемом диапазоне при записи/считывании может приводить к тому, что реальное значение данного угла может отличаться от идеального. Выше мы показали, что ошибки на этапе записи не оказывают влияния на период и угол поворота плоскостей решетки, а значит, несоответствие между расчетными и реальными значениями $\chi(i)$ будет определяться, главным образом, ошибками на этапе считывания.

Угловое расстояние между соседними лучами в „веере“ можно найти следующим образом:

$$
\chi(i)=\arccos \left[\frac{\mathbf{v}_{2}(i) \cdot \mathbf{v}_{2}(i+1)}{\left|\mathbf{v}_{2}(i)\right| \cdot\left|\mathbf{v}_{2}(i+1)\right|}\right] .
$$

Анализ показывает, что при отклонении плоскости считывания от плоскости, в которой лежат векторы всех решеток, на $0.5^{\circ}$, угловое расстояние между соседними лучами изменяется нелинейно. При этом отклонение углового расстояния от расчетного растет с номером луча и для последней пары составляет $2.1 \cdot 10^{-5 \circ}$, в то время как для первой пары лучей оно не превышает $3.32 \cdot 10^{-6 \circ}$.

Вторым важным критерием, определяющим работоспособность ГП, является плоскостность „веера“ лучей. Для ее оценки удобно использовать угол $\xi(i)$ между нормалями к плоскостям, образованным соседними лучами:

$\xi(i)=\arccos \left\{\frac{\left[\mathbf{v}_{2}(i+2) \times \mathbf{v}_{2}(i+1)\right] \cdot\left[\mathbf{v}_{2}(i+1) \times \mathbf{v}_{2}(i)\right]}{\left|\mathbf{v}_{2}(i+2) \times \mathbf{v}_{2}(i+1)\right| \cdot\left|\left[\mathbf{v}_{2}(i+1) \times \mathbf{v}_{2}(i)\right]\right|}\right\}$.

Если „веер“ идеально плоский, то такие нормали будут параллельны, и все углы $\xi(i)$ будут равны 0 (рис. 5, $a$ ).

Было установлено, что поворот образца вокруг оси $O x$ на угол $\tau$ и/или вокруг оси $O y$ на угол $\sigma$ приводит к тому, что считывающий лазерный пучок перестает лежать в плоскости „веера“ лучей. В результате следы „веера“ дифрагированных лучей на экране либо

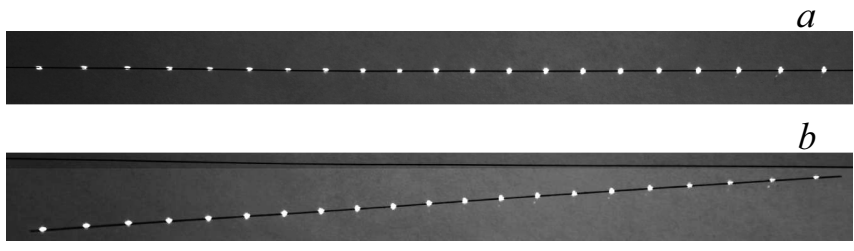

C

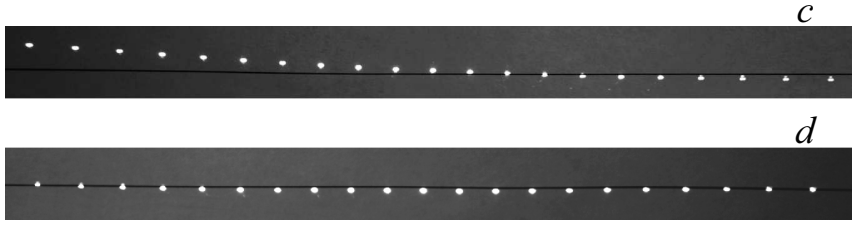

Рис. 5. Фотография следов „веера“ лучей ГП на экране: $a$ идеальный случай, $b$ - при повороте вокруг оси $O x, c-$ при повороте вокруг оси $O y, d$ - при одновременном повороте вокруг осей $O x$ и $O y$.

образуют прямую наклонную линию (рис. 5,b), либо изогнутую (рис. $5, c)$ в случае, когда углы $\xi(i)$ внутри призмы становятся ненулевыми и неравными. При одновременном повороте вокруг обеих осей описанные выше эффекты накладываются (рис. 5, d). Согласно расчетам, при повороте образца вокруг осей $O x$ и $O y$ на $2^{\circ}$ и $0.05^{\circ}$ соответственно максимальное значение угла $\xi(i)$ составит $4.875^{\circ}$.

\section{3. Влияние ошибок записи при одновременном повороте голографической призмы вокруг осей $O x$ и $O y$}

Выше была рассмотрена ситуация, когда поворот образца на этапе записи ГП для всех решеток был выполнен одинаково, однако в реальности при регистрации каждой решетки в призме из-за необходимых перемещений образца в оптической схеме ошибки его ориентации будут различны. Несмотря на то что сама величина ошибок не приводит к серьезным изменениям в периоде и угле поворота решетки, необходимо рассмотреть, как указанные ошибки повлияют на расположение векторов решеток в одной плоскости, если учесть, что у каждой решетки своя ошибка.

Рассмотрим максимальное отклонение для двух соседних и двух крайних решеток в реальной призме. При этом ошибки в установке образца при записи каждой решетки положим максимальными и противоположными по знаку, т.е. если одна решетка записывается, когда образец повернут вокруг осей $O x$ и $O y$ по часовой стрелке, то для второй решетки поворот осуществлен против часовой стрелки.

Расчеты показали, что в данном случае угол между плоскостью, образованной векторами первой и второй решеток, и плоскостью $O x y$ составляет $11.797^{\circ}$, а между 
плоскостью, образованной векторами первой и 21-й решеток, и плоскостью $O x y-14.739^{\circ}$.

Для одновременного восстановления таких голограмм считывающий луч, направленный параллельно плоскости $O x y$, должен падать на образец под углом $25.058^{\circ}$ в случае для пары из первой и второй решеток, а для пары из первой и 21-й - 25.069.

Так как различие в углах считывания по порядку величины соответствует угловой селективности отдельной решетки ГП, то при считывании такого веера должна наблюдаться разница в интенсивностях составляющих его лучей. Если различие в углах считывания будет превышать селективность решетки, то лучи веера начнут восстанавливаться по очереди, т.е. призма потеряет свою функциональность. Таким образом, можно заключить, что общая ошибка при записи отдельных решеток не должна превышать $6.103^{\circ}$ по углу $\tau$ и $1.41^{\circ}$ по углу $\sigma$.

Угол $\xi(i)$ между нормалями к плоскостям, образованным первым-вторым и первым-21-м лучами, составляет $34.638^{\circ}$. Таким образом, дифрагированные лучи „веера“ не лежат в одной плоскости, и их следы на экране образуют кривую. Угловое расстояние между первым и вторым лучами составляет $3.1977^{\circ}$, т.е. ошибка в угловом расстоянии равна $0.6977^{\circ}$, а угловое расстояние между первым и 21-м лучами - 50.1066º, т.е. общая ошибка в угловом диапазоне „веера“ составит $0.1066^{\circ}$. Таким образом, можно заключить, что угол между соседними лучами непостоянен, а величина ошибки зависит от номера луча, и если для первых лучей она положительна, то для последних - отрицательна. Углы, под которыми первый, второй и 21-й дифрагированные лучи выходят из образца, составляют $3.12,5.568$ и $53.014^{\circ}$ соответственно. То есть ошибка в этом угле для первого, второго и 21-го лучей равна 0.12, 0.068 и $0.014^{\circ}$.

\section{Выводы}

В настоящей работе проведен анализ влияния ошибок записи и считывания на рабочие характеристики ГП на 21 канал, записанной в ФТР стекле. Показано, что ошибки в установке образца в исследуемом диапазоне поворотов вокруг двух ортогональных осей при записи не оказывают влияния на периоды и углы поворота решеток внутри образца. Если все решетки записаны с одинаковым отклонением от идеального положения, то все векторы решеток лежат в одной плоскости. Однако сама плоскость может поворачиваться в среде, что приведет к изменению угла считывания и углов, под которыми дифрагированные лучи выходят из образца.

Если при записи каждой решетки призмы ошибки различны, то векторы решеток не будут лежать в одной плоскости, что может привести к потере призмой функциональности. Также различие ошибок ведет к появлению неплоскостности „веера“. Было установлено, что для сохранения функциональности ГП общая ошибка при записи отдельных решеток не должна превышать $6.103^{\circ}$ по углу $\tau$ и $1.41^{\circ}$ по углу $\sigma$.

Также показано, что даже при записи идеальной призмы ошибки на этапе считывания могут приводить к скручиванию веера и нарушению его плоскостности.

Работа выполнена при финансовой поддержке Министерства образования и науки Российской Федерации (проект 16.1651.2017/4.6).

\section{Список литературы}

[1] Грановский В.А., Кудрявцев М.Д., Рыскин А.И., Щеулин А.С. // Опт. и спектр. 2009. Т. 106. № 5. C. 822; Granovskii V.A., Kudryavtsev M.D., Ryskin A.I., Shcheulin A.S. // Opt. Spectrosc. 2009. V. 106. N 5. P. 855.

[2] Ангервакс А.Е., Грановский В.А., Кудрявцев М.Д., Рыскин А.И., Щеулин А.С. // Опт. и спектр. 2010. Т. 108. № 5. C. 871; Angervaks A.E., Granovskii V.A., Kudryavtsev M.D., Ryskin A.I., Shcheulin A.S. // Opt. Spectrosc. 2010. V. 108. N 5. P. 824.

[3] Ангервакс А.Е., Грановский В.А., Кудрявцев М.Д., Рыскин А.И., Щеулин А.С. // Опт. и спектр. 2012. Т. 112. № 2. C. 343; Angervaks A.E., Granovskii V.A., Kudryavtsev M.D., Ryskin A.I., Shcheulin A.S. // Opt. Spectrosc. 2012. V. 112. N 2. P. 312.

[4] Ангервакс А.Е., Гороховский К.С., Грановский В.А., Doan Van Bac, Иванов С.A., Окунь Р.А., Никоноров Н.В., Рыскин А.И. // Опт. и спектр. 2017. Т. 123. № 6. C. 963; Angervaks A.E., Gorokhovskii K.S., Granovskii V.A., Doan Van Bac, Ivanov S.A., Okun' R.A., Nikonorov N.V., Ryskin A.I. // Opt. Spectrosc. 2017. V. 123. N 6. P. 970.

[5] Ivanov S.A., Angervaks A.E., Doan Van Bac, Nikonorov N.V., Okun' R.A. // Proc. SPIE. 2017. V. 10329. P. $103292 Z$. doi $10.1117 / 12.2270186$

[6] Иванов С.А., Доан В., Игнатьев А.И., Никоноров Н.В. // Научно-технический вестник информационных технологий, механики и оптики. 2016. Т. 16. № 3(103). С. 428; Ivanov S.A., Doan Van Bac, Ignatiev A.I., Nikonorov N.V. // Sci. Tech. J. Inf. Technol. Mech. Opt. 2016. V. 16. N 3(103). P. 428 (in Russian).

[7] Лурье А.И. Аналитическая механика. М.: Физматлит, 1961. 824 c.; Lur'ye A.I. Analiticheskaya mekhanika. Fizmatlit, M., 1961 (in Russian).

[8] Кольер Р., Беркхарт К., Лин Л. Оптическая голография. M.: Мup, 1973. 688 c.; Collier R.J., Burckhardt C.B., Lin L.H. Optical Holography. NY.: Academic Press., 1971. 